\title{
Direct observation of surface polarization at hybrid perovskite/Au interfaces by dark transient experiments
}

Cite as: Appl. Phys. Lett. 116, 183503 (2020); https://doi.org/10.1063/5.0006409

Submitted: 02 March 2020. Accepted: 18 April 2020 . Published Online: 05 May 2020

Jorge Caram (D), Marisé García-Batlle (D), Osbel Almora (D), Roberto D. Arce (D), Antonio Guerrero (D), and Germà Garcia-Belmonte (D)

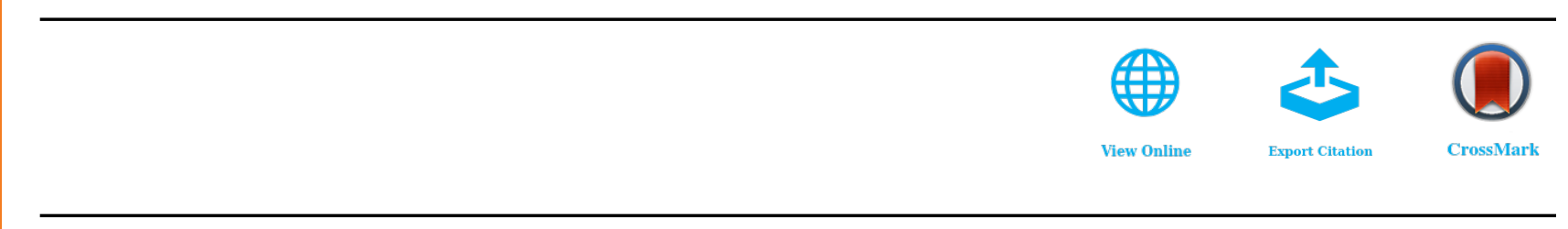

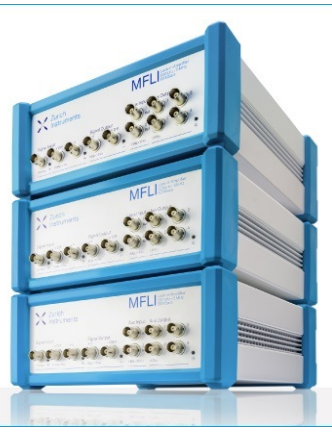

116,183503 


\title{
Direct observation of surface polarization at hybrid perovskite/Au interfaces by dark transient experiments
}

Cite as: Appl. Phys. Lett. 116, 183503 (2020); doi: 10.1063/5.0006409

Submitted: 2 March 2020 - Accepted: 18 April 2020 .

Published Online: 5 May 2020

Jorge Caram, ${ }^{1,2,3}$ iD Marisé García-Batlle, ${ }^{1}$ (D) Osbel Almora, ${ }^{1,4,5}$ (D) Roberto D. Arce, ${ }^{2,3}$ (D) Antonio Guerrero, ${ }^{1}$ and Germà Garcia-Belmonte ${ }^{1, a)}$ (iD

\author{
AFFILIATIONS \\ 'Institute of Advanced Materials (INAM), Universitat Jaume I, 12006 Castelló, Spain \\ ${ }^{2}$ Instituto de Física del Litoral (IFIS-CONICET), Güemes 3450-3000 Santa Fe, Argentina \\ ${ }^{3}$ Facultad de Ingeniería Química, UNL, Sgo. del Estero 2829-3000 Santa Fe, Argentina \\ ${ }^{4}$ Institute of Materials for Electronics and Energy Technology (i-MEET), Friedrich-Alexander-Universität Erlangen-Nürnberg, \\ 91058 Erlangen, Germany \\ ${ }^{5}$ Erlangen Graduate School in Advanced Optical Technologies (SAOT), Friedrich-Alexander-Universität Erlangen-Nürnberg, \\ 91052 Erlangen, Germany
}

${ }^{a)}$ Author to whom correspondence should be addressed: garciag@uji.es

\begin{abstract}
A distinctive feature of hybrid perovskite light-absorbing materials is the non-negligible ionic conductivity influencing photovoltaic performance and stability. Moving ions or vacancies can naturally accumulate at the outer interfaces (electrode polarization) upon biasing. Contrary to that approach, a modulation of conductive or recombination properties could manifest as an alteration in the low-frequency part of the impedance response, either producing inductive or large capacitive features. Under this last view, capacitances are not the response of polarized structures or charging mechanisms, but result from the modulation of currents. This work intends to provide pieces of evidence that assist us in distinguishing between these two dissimilar mechanisms, namely, real charge polarization and delayed current effects under bias in the dark. The analysis relays upon an experimental technique based on transient charging signals using the Sawyer-Tower circuit. Instead of applying an alternating small perturbation over a steady-state voltage (differential capacitance method), transient charging measures the resulting polarization upon a large bias step under the suppression of dc currents. Our findings reveal that real steady-state charge is indeed induced by the applied voltage in the dark, easily interpreted by means of charged real capacitors with values much larger than the geometrical capacitance of the film. The connection between that polarization and the charging of perovskite/contact interfaces is highlighted.
\end{abstract}

Published under license by AIP Publishing. https://doi.org/10.1063/5.0006409

In just a few years, perovskite solar cells (PSCs) have gained great attention in the field of photovoltaics with an unprecedented increase in power conversion efficiency (PCE) achieved from facile solution processing routes. ${ }^{1-4}$ Despite those technological progresses, it was identified from the very beginning that slow responses in PSCs cause annoying deviations from common photovoltaic operation. The presence of slow relaxation processes (from milliseconds to minutes) yield different hysteretic $J-V$ curves depending on the bias sweep direction, scan rate, illumination, and poling history. ${ }^{2,5-7}$ That phenomenon was initially connected to the observation of an excess capacitive signal in the low frequency part of the capacitance spectra, ${ }^{8,9}$ in relation to the polarization of the outer contacts upon bias which originates the so-called dark hysteresis. By reinforcing the idea of interfacial charging under solar cell operation, several studies identified the formation of built-in potentials in the vicinity of the interface between the perovskite absorber and extracting layers. ${ }^{10}$ Using frequency-modulation Kelvin probe force microscopy (FM-KPFM), it has been identified that the electrical field in solar cells under working conditions mainly drops at the corresponding selective interfaces by local charge accumulation. ${ }^{11} \mathrm{~A}$ related phenomenon is the remnant open-circuit voltage commonly exhibited in long-time photovoltage decay experiments, ${ }^{12}$ which has also been explained in terms of the piling up of stabilized interfacial charge. 
A distinctive feature of perovskite light-absorbing materials is the non-negligible ionic conductivity that is believed to influence photovoltaic operation. ${ }^{13,14}$ Moving ions or vacancies naturally accumulate at the outer interfaces (electrode polarization) upon biasing, ${ }^{15,16}$ originating the usually reported excess dark capacitance of the order $1-10 \mu \mathrm{F} \mathrm{cm}^{-2}$. Similar to the accumulation of ionic species, interfacial electronic pileup has been proposed in order to interpret the commonly reported light-enhanced capacitance at low frequencies. ${ }^{17}$ Accordingly, recent models can be found on the interplay of electronic carrier recombination and moving ion dynamics. ${ }^{18,19}$

Previous studies ${ }^{20}$ suggested a close dependence between the ionic environment and the recombination mechanism in PSCs, in such a way that ionic vacancies move to decrease the recombination flux. It has also been argued ${ }^{21}$ that such a coupling effect between ionic and electronic charges has an influence at low frequencies, as the carrier recombination current becomes phase-delayed with respect to the voltage perturbation. Under this view, the observed low-frequency light-enhanced capacitance is caused by out-of-phase carrier recombination instead of charge accumulation currents. Sophisticated equivalent circuits (ionically gated transistor interface) have been proposed ${ }^{22}$ accounting for the ionic-electronic interplay governing charge injection and recombination. Similar arguments have appeared very recently disregarding ionic effects and highlighting delayed electronic injection currents as the sole cause for the low-frequency capacitive phenomena even in the dark. ${ }^{23}$ These kind of models are very rich as they introduce a modulation of conductive or recombination properties that ultimately manifests as an alteration in the low-frequency part of the impedance response. ${ }^{24}$ Their uses are well-known in electronics. For example, the conductivity modulation of $p-n$ junction diodes at a forward bias produces inductive loops, ${ }^{25}$ or the thermal diffusivity of thermoelectric modules gives rise to large low-frequency capacitances. $^{26}$

As a consequence, it is still under debate whether the large capacitive effects observed at low-frequencies in PSCs are exclusively explained from charge accumulation mechanisms (interface polarization) or, instead, a dynamic interplay between ionic kinetics and electronic carrier recombination/injection current occurs giving rise to apparent (not real) capacitors. Our work intends to provide pieces of evidence that assist us in distinguishing between real charge polarization and delayed current effects under bias stimuli in the dark. This distinction is crucial for understanding the basic functioning and device operation of PSCs.

Here, we select perovskite films of methylammonium lead iodide $\left(\mathrm{MAPbI}_{3}\right)$ as the active layer, symmetrically contacted by interdigitated gold electrodes (see below for the experimental conditions). This experimental setup avoids the formation of a photovoltage under light irradiation, which is expected when asymmetrical (different work function) materials are used as selective contacting layers. One can observe in Fig. 1 the impedance and capacitance responses registered at zero-bias in the dark and ambient conditions, exhibiting two main features: at high- and intermediate-frequencies $(f>100 \mathrm{~Hz})$, the geometrical capacitance $C_{g} \approx 2.7 \mathrm{pF}$ and sample resistance dominate; and at lowfrequencies, the commonly reported excess capacitance increment $C_{s} \gg C_{g}$ is observed. The same electrical response is observed for $1 \mathrm{~V}$-bias (Fig. S1) and corresponds to the equivalent circuit shown in Fig. S2. An estimation of the effective polarization area of the interdigitated electrodes is included as the supplementary material, which a)

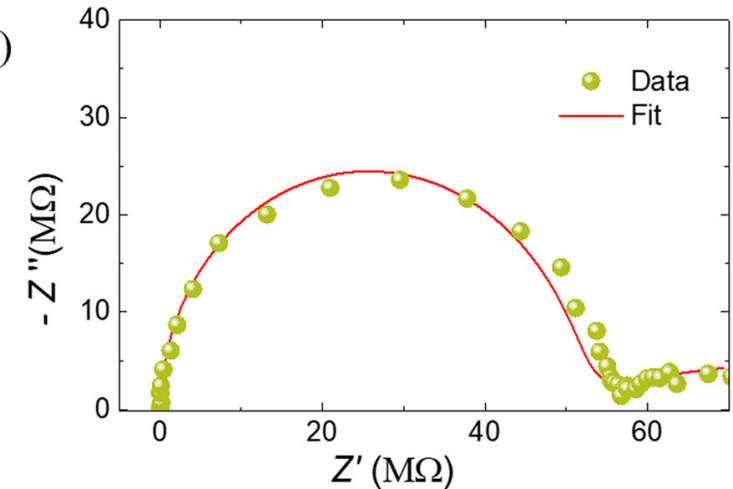

b)

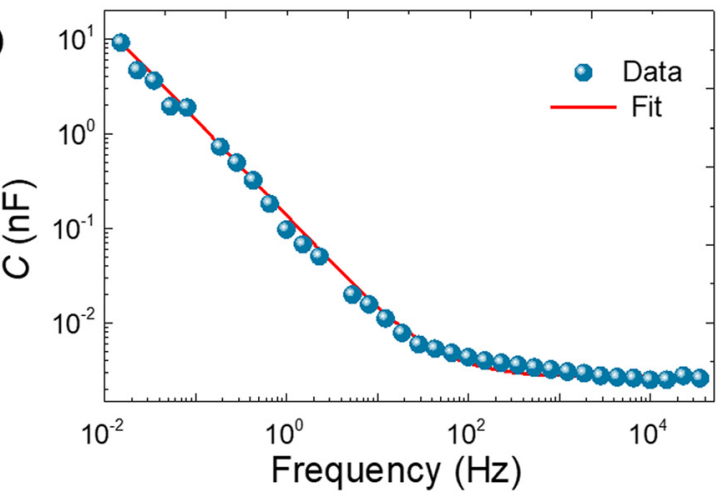

FIG. 1. (a) Example of impedance plot and (b) capacitance spectrum response of a $320 \mathrm{~nm}$-thick $\mathrm{MAPbl}_{3}$ film, measured at zero bias in the dark, exhibiting lowfrequency capacitive features. In both graphs, the red lines represent a fitting with the corresponding equivalent circuit (FIG. S2).

results in values of the geometrical capacitance per unit area of $C_{g} \approx$ $0.1 \mathrm{nF} \mathrm{cm}^{-2}$.

Because small-amplitude capacitive analysis by itself is not able to unambiguously discern if the low-frequency capacitance is effectively caused by charge polarization, we propose here to rely upon a different experimental technique based on transient charging signals. Instead of applying an alternating small perturbation over a steady-state voltage (differential capacitance method), transient charging measures the resulting polarization upon a large bias step under the suppression of $\mathrm{dc}$ currents. The experimental setup is based on the Sawyer-Tower circuit $(\mathrm{ST})^{27}$ which is the classical setup used to explore the hysteretic loops of ferroelectric materials. The configuration of the circuit is shown in Fig. 2(a). A step voltage signal ( $\left.V_{\text {app }}\right)$ is applied to the sample $C_{x}$ across a reference linear capacitor $C_{0}$, with both capacitors connected in series. The condition $C_{0} \gg C_{x}$ must be accomplished to assure that the applied voltage principally drops within the perovskite sample. Under this condition, the charge storage in $C_{x}$ (as in $C_{0}$ ) is $Q=C_{0} V_{0}$, with $V_{0}$ being the voltage at the reference capacitor ([see Fig. 2(b)], which is recorded with an ultrahigh input resistance (1 T $\Omega$ ) potentiostat to avoid loading effects from the recording instrument. The total transfer function of the measuring setup is given in the supplementary material.

As observed in Fig. 2(b), the voltage at $C_{0}$ follows the bias perturbation steps in such a way that only a small portion drops at it, i.e., $V_{0} \ll V_{\text {app }}$. Note that this fact discards the observation of the 

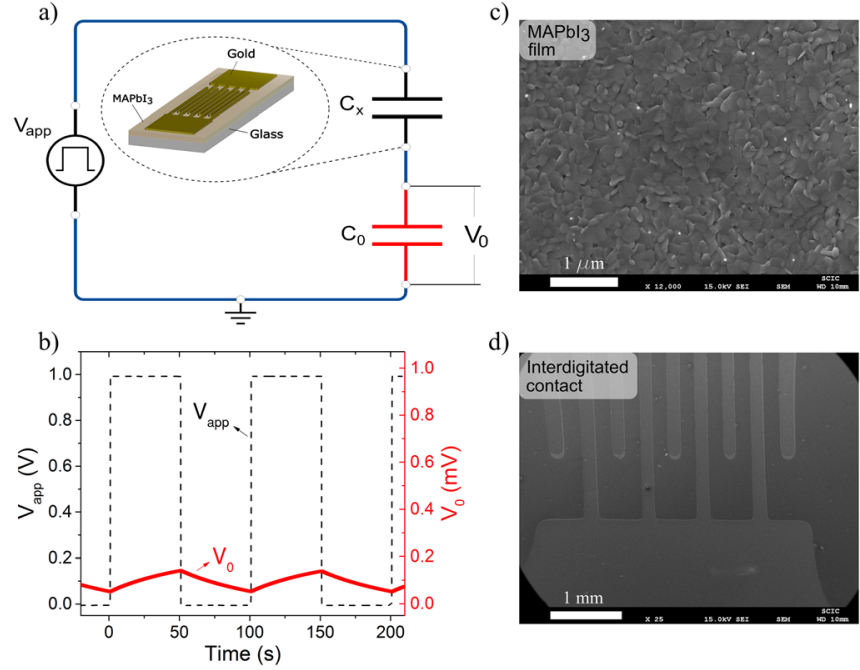

d)

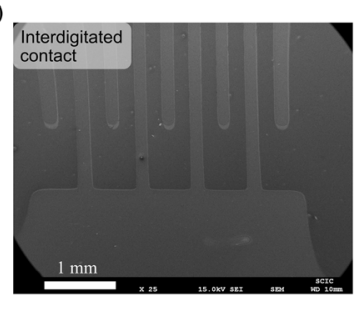

FIG. 2. (a) Sawyer-Tower circuit for charge transient measurements. (b) Example of the charge transient response of a $320 \mathrm{~nm}$-thick $\mathrm{MAPbl}_{3}$ film registered in the dark conditions. The applied rectangular voltage pulse of $1 \mathrm{~V}$-height is compared with the induced voltage $V_{0}$ (note the scale differences). (c) SEM image of the top view of an $\mathrm{MAPbl}_{3}$ film. (d) SEM image of the perovskite interdigitated (ID) contacts.

reference capacitor $C_{0}$ charging through the perovskite sample shunt resistance, which would yield $V_{0}=V_{\text {app }}$ in the long time (see more in the discussion below). To verify the effect of the perovskite film on the reported polarization, the setup response comprising only gold electrodes on glass substrates was checked (see Fig. S3). Since the reference capacitor suppresses any dc current, a registered steady-state voltage after long-enough poling should correspond to the effect of a true charging mechanism. Otherwise, the reference capacitor cannot withstand constant polarization. A delayed or modulated injection current is not able to yield a permanent voltage on the reference capacitor plate. On the contrary, a measured potential at $C_{0}$ should indicate a kind of locally imbalanced charge at the perovskite sample.

All materials in our experiments were used as received: $\mathrm{CH}_{3} \mathrm{NH}_{3} \mathrm{I}$ (DYESOL), $\mathrm{PbI}_{2}$ (TCI, 99.99\%), N, N-dimethylformamide anhydrous (Sigma Aldrich, 99.8\%), dimethylsulfoxide anhydrous (Sigma Aldrich, $\geq 99.9 \%$ ), and chlorobenzene anhydrous (Sigma Aldrich, 99.8\%). The perovskite precursor solution was obtained from reacting dimethylformamide (DMF) solutions (50 wt. \%) containing MAI and $\mathrm{PbI}_{2}$ (1:1 mol. \%) and MAI, $\mathrm{PbI}_{2}$, and DMSO (1:1:1 mol. \%). Perovskite layers were deposited on the top of $25 \times 25 \mathrm{~mm}^{2}$ glass by spin-coating at $4000 \mathrm{rpm}(4000 \mathrm{ac})$ for $50 \mathrm{~s}$. Chlorobenzene was used as an antisolvent and added just before the white solid begins to crystallize in the substrate. A mask with an interdigitated (ID) pattern (9 digits, $0.2 \mathrm{~mm}$ between digits, $0.3 \mathrm{~mm}$ width, and $7.8 \mathrm{~mm}$ length) was used. Gold was thermally evaporated $(100 \mathrm{~nm})$ at a base pressure of $6 \times 10^{-6}$ mbar. The films were characterized by UV-Vis absorption spectroscopy in a Cary 500 Scan VARIAN spectrophotometer (250-900 nm), obtaining distinctive spectra with the corresponding absorption edge at $780 \mathrm{~nm}$ for $\mathrm{MAPbI}_{3}$ with a bandgap of $1.60 \mathrm{eV}$ (see Fig. S4). A mechanic Dektak 6M profilometer (Veeco) was used for film thickness measurement and the values were optimized by changing the spinning speed

(see Fig. S5). Also, XRD patterns of $\mathrm{MAPbI}_{3}$-films are included in Fig. S8. SEM analysis also shows the polycrystalline nature of the perovskite [Fig. 2(c)] and the ID geometric configuration [Fig. 2(d)] of the contacts. The examination of different thicknesses allows obtaining the optimum values (see Fig. S5). Direct impedance measurements as shown in Figs. 1 and S1 were carried out by using a PGSTAT-30 Autolab potentiostat equipped with an impedance module. Also, ST transients, as in Fig. 2(b), were recorded with the same potentiostat. All electrical experiments were performed at room temperature in the air.

Figure 3(a) shows a representative transient response, using the setup in Fig. 2(a), occurring in an $\mathrm{MAPbI}_{3}$ perovskite film of $320 \mathrm{~nm}$ thickness with an ID contact configuration. Here, reference capacitors within the range of $C_{0}=10-100 \mu \mathrm{F}$ were employed, which were previously checked using impedance analysis (see Fig. S6), and showed a plateau at the frequencies of interest $(f<1 \mathrm{kHz})$. By examining Fig. 3(a), one can observe charging signals upon application of a step bias of height $V_{\text {app }}=1 \mathrm{~V}$ in the dark conditions. A necessary test to check experimental consistency consists of verifying that $V_{0} \ll V_{\text {app }}$. As observed in Fig. 3, $V_{0}$ inversely scales with the reference capacitor with values (in the $\mathrm{mV}$ range) much lower than the bias $(1 \mathrm{~V})$, in good accordance with the assumption $Q=C_{0} V_{0}$ (see the transfer function calculation in the supplementary material). This fact precludes the possibility of seeing the charging of the reference capacitor through the perovskite sample shunt resistance with time constant

a)

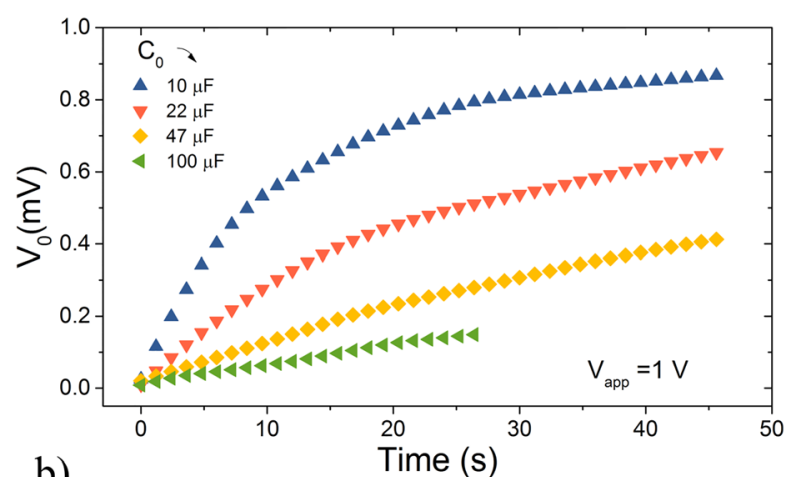

b)

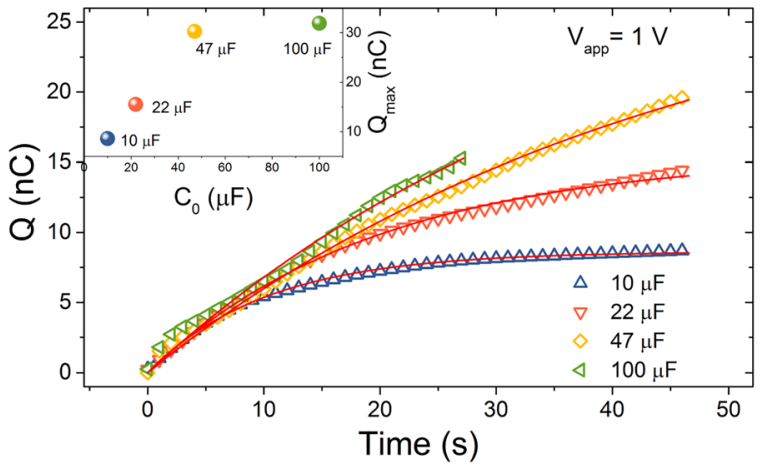

FIG. 3. (a) Polarization voltage signals for $\mathrm{MAPbl}_{3}$ films with $1 \mathrm{~V}$ applied bias in the dark conditions. The reference capacitor ranges $C_{0}=10-100 \mu \mathrm{F}$, as indicated. (b) Charge transients. In the inset: $Q_{\max }$ induced for long-time poling using different reference capacitors, calculated from fitting to an exponential function. 
$\tau=R_{s h} C_{0} \approx 10^{3}-10^{4} \mathrm{~s}$, which would yield a saturation of the registered voltage as $V_{0} \approx V_{\text {app }}$ at long time polarization. Contrary to this response, a rather steady-state voltage (charge) is attained (accumulated) for $\sim 50$ s-poling. This is particularly visible for $C_{0}=10 \mu \mathrm{F}$.

The total amount of charge, calculated by using exponential fitting, attains values within the range of $Q_{\max } \approx 10-30 \mathrm{nC}$ [inset of Fig. 3(b)]. Remarkably, all curves collapse in the initial ( $\sim 10 \mathrm{~s})$ part of the transient, thus reinforcing our claim that $C_{0}$ charges as a consequence of the perovskite interface polarization and not through the shunt resistance. The reported dispersion in the total accumulated charge can be understood in terms of the ionic and, presumably, reactive character of the polarization mechanism occurring at the interfaces (see below).

It is remarkable that the amount of charge does not match that expected from the polarization of the perovskite bulk. As inferred from Fig. 1(b), the dielectric properties of the films give rise to a geometrical capacitance $C_{g} \approx 2.7 \mathrm{pF}$, corresponding to the highfrequency plateau of the capacitance spectra. A value that would entail polarization charges of the order of $\mathrm{pC}$ (given $1 \mathrm{~V}$-bias) as $Q \approx C_{g} V_{\text {app }}$. Such a small value is in contradiction to those actually encountered within the order of $10 \mathrm{nC}$ [Fig. 3(b)]. Instead, the amount of charge actually correlates with the values attained by the lowfrequency capacitance in Fig. 1(b): $C \approx 2 \mathrm{nF}$ at $f=0.1 \mathrm{~Hz}$.

By comparing capacitive and transient charging experiments, we can infer that the same mechanism in behind both responses. That mechanism manifests either as a low-frequency excess capacitance or as a steady-state charge induced by the applied voltage. The important difference between these two measuring techniques is that while impedance gives a differential, alternating-current small-amplitude response, transient charging is a large-amplitude procedure yielding in our experiments a permanent charge only explainable if true polarization occurs. Therefore, a modulated or delayed injection current cannot be claimed as originating that observed steady-state response, simply because dc current is suppressed in the used setup. If the lowfrequency excess capacitance was an apparent response, one would have expected polarization charges only caused by dielectric properties $Q \approx C_{g} V_{\text {app }}$, in opposition to that observed here.

The occurrence of polarized interfaces in PSCs was proposed some years ago. ${ }^{9}$ In the dark, the corresponding excess capacitance has been connected to the formation of double layer-like structures in the vicinity of the perovskite/contact interface in which mobile ions are piled up (Fig. 4) and shield the part of the applied voltage. ${ }^{16,28}$ Excess

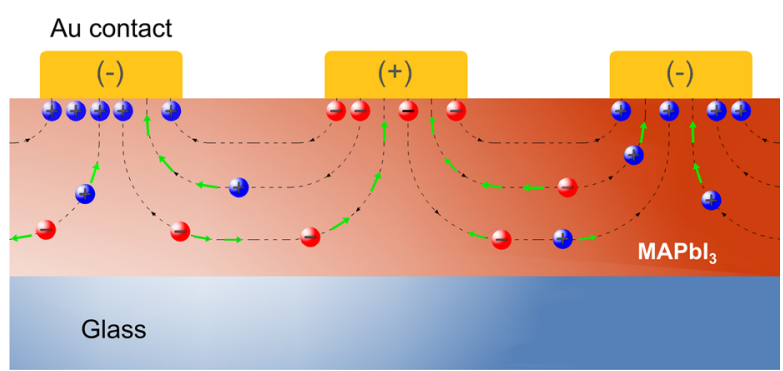

FIG. 4. The process of interfacial polarization is schematized. lonic mobile charges are driven by the applied electric field (dashed line) toward the contacts, causing a real polarization/accumulation of charge at the interface between $\mathrm{Au}$ contacts and $\mathrm{MAPbl}_{3}$ film dark capacitance of the order $1-10 \mu \mathrm{F} \mathrm{cm}^{-2}$ can be readily explained by this way. We can even roughly estimate the concentration of mobile ions contributing to the surface polarization at the outer electrodes. The perovskite total volume of our samples can be calculated (see Fig. S7) as $\sim 10^{-5} \mathrm{~cm}^{3}$, which gives rise to mobile ionic densities of the order $N \approx 10^{16} \mathrm{~cm}^{-3}$, which corresponds to the density portion effectively contributing to the interface polarization. That value is in agreement with recent and previous estimations. ${ }^{29}$

Apart from purely electrostatic views accounting for the interface polarization, it has been also recognized that chemical interactions between mobile ions, photogenerated electronic carriers, and contacting materials might give rise to the formation of dipole-like structures. ${ }^{30,31}$ A survey about the chemical reactivity of the perovskite/ contact materials can be found elsewhere. ${ }^{28}$ It has been emphasized by Bisquert and co-workers in their review paper that the kinetics of charging is hardly understood exclusively in terms of diffusion and double-layer formation. Moreover, interface reactivity forming local chemical bonds largely influences overall the resulting electronic and ionic dynamics. The complexity and lack of complete reproducibility inferred from the dispersion in the total accumulated charge of Fig. 3(b) should be related to the known chemical interaction between $\mathrm{MAPbI}_{3}$ and gold electrodes, as recently highlighted. ${ }^{30}$

We remark that our findings corroborate the occurrence of real polarization (charge imbalance) taking place in perovskite absorbers upon bias in the dark. On the contrary, several papers have recently proposed $^{21-23}$ that the commonly reported low-frequency capacitance features relate to the modulation of operating currents, either recombining or injecting currents, that evolve during the measurement. They claim that this effect creates out-of-phase signals not originated by charge polarization and wrongly interpreted in terms of the response of true capacitors. Following this view, it has also been claimed that measured capacitances are just apparent ${ }^{23}$ and not connected to any carrier pileup or space charge formation, even under dark conditions. ${ }^{23}$ Obviously, that explanation should be confronted with additional experiments, and not only with impedance responses. Here, we have demonstrated the appearance of steady-state polarization voltages in the dark, easily interpreted by means of charged capacitors with values much larger than the geometrical capacitance of the film. We connect that polarization to the charging of perovskite/contact interfaces by mobile ions.

See the supplementary material for additional information on sample preparation and setup checking and transfer function calculation.

We acknowledge the support from the Horizon 2020 project (Grant No. 871336-PEROXIS). M.G.-B. acknowledges Generalitat Valenciana for Grant No. GRISOLIAP/2018/073. SCIC at UJI are also acknowledged. O.A. acknowledges the financial support from the VDI/VD Innovation + Technik GmbH (Project-title: PV-ZUM) and the SAOT funded by the German Research Foundation (DFG) in the framework of the German excellence initiative. J.C. acknowledges the partial support by project PUE-054-CONICET, Argentina.

Data available on request from the authors.

\section{REFERENCES}

${ }^{1}$ J. Bisquert, E. J. Juárez-Pérez, and P. V. Kamat, Hybrid Perovskite Solar Cells: The Genesis and Early Developments 2009-2014 (Fundació Scito, Valencia, 2017), p.150. 
${ }^{2}$ M. A. Green, A. Ho-Baillie, and H. J. Snaith, Nat. Photonics 8(7), 506 (2014). ${ }^{3}$ N.-G. Park, M. Grätzel, T. Miyasaka, K. Zhu, and K. Emery, Nat. Energy 1, 16152 (2016).

${ }^{4}$ Y.-K. Ren, X.-H. Ding, Y.-H. Wu, J. Zhu, T. Hayat, A. Alsaedi, Y.-F. Xu, Z.-Q. Li, S.-F. Yang, and S.-Y. Dai, J. Mater. Chem. A 5(38), 20327 (2017).

${ }^{5}$ E. L. Unger, E. T. Hoke, C. D. Bailie, W. H. Nguyen, A. R. Bowring, T. Heumüller, M. G. Christoforod, and M. D. McGehee, Energy Environ. Sci. 7, 3690 (2014).

${ }^{6}$ N. J. Jeon, J. H. Noh, Y. C. Kim, W. S. Yang, S. Ryu, and S. I. Seok, Nat. Mater. 13, 897 (2014).

${ }^{7}$ R. S. Sanchez, V. Gonzalez-Pedro, J.-W. Lee, N.-G. Park, Y. S. Kang, I. MoraSero, and J. Bisquert, J. Phys. Chem. Lett. 5, 2357-2363 (2014).

${ }^{8}$ E. J. Juarez-Perez, R. S. Sanchez, L. Badia, G. Garcia-Belmonte, Y. S. Kang, I. Mora-Sero, and J. Bisquert, J. Phys. Chem. Lett. 5(13), 2390 (2014).

${ }^{9}$ O. Almora, I. Zarazua, E. Mas-Marza, I. Mora-Sero, J. Bisquert, and G. GarciaBelmonte, J. Phys. Chem. Lett. 6(9), 1645 (2015).

${ }^{10}$ V. W. Bergmann, Y. Guo, H. Tanaka, I. M. Hermes, D. Li, A. Klasen, S. A. Bretschneider, E. Nakamura, R. Berger, and S. A. L. Weber, ACS Appl. Mater, Interfaces 8(30), 19402 (2016).

${ }^{11}$ S. A. L. Weber, I. M. Hermes, S.-H. Turren-Cruz, C. Gort, V. W. Bergmann, L. Gilson, A. Hagfeldt, M. Graetzel, W. Tress, and R. Berger, Energy Environ. Sci. 11(9), 2404 (2018).

${ }^{12}$ R. Gottesman, P. Lopez-Varo, L. Gouda, J. A. Jimenez-Tejada, J. Hu, S. Tirosh, A. Zaban, and J. Bisquert, Chem 1(5), 776 (2016).

${ }^{13}$ R. Gottesman, E. Haltzi, L. Gouda, S. Tirosh, Y. Bouhadana, A. Zaban, E. Mosconi, and F. De Angelis, J. Phys. Chem. Lett. 5(15), 2662 (2014).

${ }^{14}$ W. Tress, N. Marinova, T. Moehl, S. M. Zakeeruddin, M. K. Nazeeruddin, and M. Grätzel, Energy Environ. Sci. 8(3), 995 (2015).

${ }^{15}$ R. A. Belisle, W. H. Nguyen, A. R. Bowring, P. Calado, X. Li, S. J. C. Irvine, M. D. McGehee, P. R. F. Barnes, and B. C. O’Regan, Energy Environ. Sci. 10(1), 192 (2017).

${ }^{16}$ O. Almora, A. Guerrero, and G. Garcia-Belmonte, Appl. Phys. Lett. 108(4), 043903 (2016)
${ }^{17}$ I. Zarazua, G. Han, P. P. Boix, S. Mhaisalkar, F. Fabregat-Santiago, I. MoraSeró, J. Bisquert, and G. Garcia-Belmonte, J. Phys. Chem. Lett. 7(24), 5105 (2016).

${ }^{18}$ S. Ravishankar, O. Almora, C. Echeverría-Arrondo, E. Ghahremanirad, C. Aranda, A. Guerrero, F. Fabregat-Santiago, A. Zaban, G. Garcia-Belmonte, and J. Bisquert, J. Phys. Chem. Lett. 8(5), 915 (2017).

${ }^{19}$ O. Almora, P. Lopez-Varo, K. T. Cho, S. Aghazada, W. Meng, Y. Hou, C. Echeverría-Arrondo, I. Zimmermann, G. J. Matt, J. A. Jiménez-Tejada, C. J. Brabec, M. K. Nazeeruddin, and G. Garcia-Belmonte, Sol. Energy Mater. Sol. Cells 195, 291 (2019).

${ }^{20}$ A. Pockett, G. E. Eperon, N. Sakai, H. J. Snaith, L. M. Peter, and P. J. Cameron, Phys. Chem. Chem. Phys. 19(8), 5959 (2017).

${ }^{21}$ D. A. Jacobs, H. Shen, F. Pfeffer, J. Peng, T. P. White, F. J. Beck, and K. R. Catchpole, J. Appl. Phys. 124(22), 225702 (2018).

${ }^{22}$ D. Moia, I. Gelmetti, P. Calado, W. Fisher, M. Stringer, O. Game, Y. Hu, P. Docampo, D. Lidzey, E. Palomares, J. Nelson, and P. R. F. Barnes, Energy Environm. Sci. 12, 1296 (2019).

${ }^{23}$ F. Ebadi, N. Taghavinia, R. Mohammadpour, A. Hagfeldt, and W. Tress, Nat. Commun. 10(1), 1574 (2019).

${ }^{24}$ M. Ershov, H. C. Liu, L. Li, M. Buchanan, Z. R. Wasilewski, and A. K. Jonscher, IEEE Trans. Electron Devices 45(10), 2196 (1998).

${ }^{25}$ J. J. H. van den Biesen, Solid-State Electron. 33(11), 1471 (1990).

${ }^{26}$ J. García-Cañadas and G. Min, J. Electron. Mater. 43(6), 2411 (2014).

${ }^{27}$ C. B. Sawyer and C. H. Tower, Phys. Rev. 35(3), 269 (1930).

${ }^{28} \mathrm{H}$. Wang, A. Guerrero, A. Bou, A. M. Al-Mayouf, and J. Bisquert, Energy Environ. Sci. 12(7), 2054 (2019).

${ }^{29}$ L. Bertoluzzi, C. C. Boyd, N. Rolston, J. Xu, R. Prasanna, B. C. O’Regan, and M. D. McGehee, Joule 4, 109 (2020).

${ }^{30}$ J. Pospisil, A. Guerrero, O. Zmeskal, M. Weiter, J. J. Gallardo, J. Navas, and G. Garcia-Belmonte, Adv. Funct. Mater. 29(32), 1900881 (2019).

${ }^{31}$ J. Carrillo, A. Guerrero, S. Rahimnejad, O. Almora, I. Zarazua, E. Mas-Marza, J. Bisquert, and G. Garcia-Belmonte, Adv. Energy Mater. 6(9), 1502246 (2016). 